\title{
High Dose Prednisolone Lowers Plasma Glycated Albumin Levels Compared to Actual Glycemic Control: A Retrospective Observational Study
}

\author{
Masami Mizuno - Katsumi Iizuka (D) - Takuma Ishihara • \\ Shusaku Fukaya $\cdot$ Shunji Yoshida $\cdot$ Jun Takeda
}

Received: October 29, 2018 / Published online: December 13, 2018

(C) The Author(s) 2018

\section{ABSTRACT}

Introduction: Glycated hemoglobin (A1c) and glycated albumin (GA) are often used as indicators of glycemic control. In this study, we determined whether prednisolone (PSL) administration lowers plasma GA.

Methods: We investigated the factors affecting GA using multivariate analysis in 48 subjects with connective tissue diseases (CTDs).

Results: Multiple regression analysis of GA showed that the dose of PSL $[\beta=-1.36 ; 95 \%$ confidence interval (CI) -2.59 to -0.14 ;

Enhanced Digital Features To view enhanced digital features for this article go to https://doi.org/10.6084/ m9.figshare.7410818.

M. Mizuno $\cdot$ S. Fukaya $\cdot$ S. Yoshida

Division of Rheumatology, Department of Internal

Medicine, Fujita Health University School of

Medicine, Toyoake, Aichi 470-1192, Japan

M. Mizuno $\cdot$ K. Iizuka $(\bowtie) \cdot J$. Takeda

Department of Diabetes and Endocrinology, Graduate School of Medicine, Gifu University, Gifu 501-1194, Japan

e-mail: kiizuka@gifu-u.ac.jp

K. Iizuka

Gifu University Hospital Center for Nutritional

Support and Infection Control, 1-1 Yanagido,

Gifu 501-1194, Japan

T. Ishihara

Gifu University Hospital Innovative and Clinical

Research Promotion Center, 1-1 Yanagido,

Gifu 501-1194, Japan $p=0.03]$, age $(\beta=0.06 ; \quad 95 \%$ CI $0.03-0.09$; $p<0.001)$, body mass index (BMI) $(\beta=-0.14$; $95 \% \mathrm{CI}-0.28$ to $-0.01 ; p=0.042)$, and A1c $(\beta=1.4 ; 95 \%$ CI $0.38-2.42 ; p=0.008)$ significantly correlated with GA (adjusted $R^{2}=0.518$ ). Moreover, GA levels adjusted for age, sex, BMI, plasma albumin (Alb) and creatinine (Cre), and A1c in the subjects taking $\geq 5 \mathrm{mg}$ PSL was significantly lower than those in those taking $<5 \mathrm{mg}$ PSL. Finally, the dose of PSL (as a continuous variable) was negatively correlated with GA adjusted for age, sex, BMI, Alb, Cre, and A1c. Conclusion: High dose ( $\geq 5 \mathrm{mg}$ ) PSL reduces GA concentration more than glycemia.

Keywords: Connective tissue diseases; Glycated albumin; Glycated hemoglobin; Prednisolone

\section{INTRODUCTION}

Glucocorticoids are widely used for the treatment of diseases such as autoimmune disease and chronic kidney disease [1, 2]. However, such treatment predisposes to diabetes, with odds ratios for new-onset diabetes mellitus (DM) in patients treated with glucocorticoids having been shown to be $1.5-2.5[1,2]$. Steroidinduced DM is associated with marked postprandial hyperglycemia due to peripheral insulin resistance and islet cell dysfunction [1]. Insulin sensitizers, such as thiazolidinediones 
and metformin, are often used as first-line therapies. However, insulin secretory capacity is also reduced; therefore, insulin therapy and oral hypoglycemic drugs may also be used [3]. Following these steps, if glycemic control remains insufficient, intensive insulin therapy may also be required after steroid therapy [3]. Early detection of steroid-induced DM is needed to prevent insulin therapy [3].

Several indices are now used to evaluate glycemic control, but glycated hemoglobin A1c (A1c) is the most frequently used. However, in specific conditions, such as hemolytic anemia and hemoglobinopathies, there are discrepancies between actual glycemic control and A1c concentration $[4,5]$. In such instances, measurement of glycated albumin (GA) is recommended [4]. Because albumin (Alb) has a shorter half-life than hemoglobin, GA reflects the efficacy of glycemic control over a shorter period of time than A1c [6], and more accurately reflects postprandial than fasting hyperglycemia [7]. Because early stage steroid-induced DM exhibits postprandial hyperglycemia with normal fasting glucose levels [1], GA can be an appropriate indicator of glycemic status in patients with steroid-induced DM. However, in some previous studies, glucocorticoid administration has been shown to lower plasma GA more effectively than glycemia [8]. Nevertheless, it remains unclear whether glucocorticoid treatment reduces plasma GA concentration.

In this study, we determined whether high dose prednisolone (PSL) affects plasma GA concentration in patients with connective tissue diseases (CTDs) using multivariate analysis. The identification of factors affecting GA concentration may define contraindications for the use of GA in subjects undergoing steroid treatment.

\section{METHODS}

\section{Study Subjects}

We retrospectively reviewed the medical records of outpatients with CTDs who had attended our department at Gifu University Hospital between April 2009 and June 2016 (Table 1). Forty-eight
Table 1 Summary of connective tissue diseases

\begin{tabular}{ll}
\hline & $n=\mathbf{4 8}$ \\
\hline Rheumatoid arthritis & 6 \\
Polymyalgia rheumatica & 5 \\
Scleroderma & 5 \\
Systemic lupus erythematoses & 6 \\
Mixed connective tissue disease & 2 \\
Dermatomyositis & 3 \\
Ankylosing spondylitis & 2 \\
Autoimmune pancreatitis & 2 \\
Neuromyelitis optica & 1 \\
Sjogren syndrome & 2 \\
Microscopic polyangitis & 1 \\
Pemphigus & 2 \\
Sarcoidosis & 2 \\
Pemphigoid & 1 \\
Interstitial pneumonia & 1 \\
Behcet disease & 1 \\
Granulomatosis with polyangiitis & 2 \\
Polymyositis & 1 \\
Psoriasis vulgaris & 2 \\
\hline & \\
\hline &
\end{tabular}

subjects were identified and placed into two groups: those who had been taking $<5 \mathrm{mg}$ PSLequivalent per day, and those who had been taking $\geq 5 \mathrm{mg}$ PSL-equivalent per day. Subjects eligible for this study met the following criteria: (1) age 20-80 years; (2) dose of steroid unchanged within a 3-month period; and (3) HbA1c concentration changed by $\leq 0.2 \%$ over a 2-month period. The exclusion criteria were as follows: (1) type $1 \mathrm{DM}$; or (2) presence of infectious disease, thyroid disease, malignant tumor, liver cirrhosis, unstable DM, anemia, or chronic renal disease (estimated glomerular filtration rate $<60 \mathrm{ml} / \mathrm{min} / 1.73 \mathrm{~mm}^{2}$, proteinuria, or hematuria). The study protocol was approved by the ethics committee of our university (Approval No. 28-139, approval date 
2016/8/3) and was designed in accordance with the Declaration of Helsinki. This study did not involve an intervention, and was retrospective and observational in nature, and therefore did not require a trial registration ID.

\section{Measurement of A1c and GA}

Plasma A1c was measured using an automated high-performance liquid chromatography analyzer (HLC-723-G9; Tosoh Corporation, Tokyo, Japan) and is presented as a National Glycohemoglobin Standardization Program value. GA concentration was measured using an enzymatic method involving albumin-specific proteinase, ketoamine oxidase, and albumin assay reagent (Lucica GA-L; Asahi Kasei Pharma Corp., Tokyo, Japan).

\section{Statistical Methods}

Using a Monte Carlo simulation, we determined that 48 patients would be sufficient to detect a mean reduction of 0.2 in GA per unit of PSL with an SD of 2.4, 5 in SD of PSL, 82\% power, and an $\alpha$ level of 0.05 . Patients with CTDs were divided into two treatment groups, those who had been taking $<5 \mathrm{mg}$ and those who had been taking $\geq 5 \mathrm{mg}$ PSL per day. Continuous variables are represented as median, 25th and 75 th percentile. Categorical variables are presented as numbers and percentages. Normally distributed clinical characteristics [age, hemoglobin $(\mathrm{Hb})$, plasma Alb and creatinine (Cre), and body mass index (BMI)] were compared using Student's $t$ test. A multivariable linear regression model was used to evaluate the relationship between the dose of PSL $(<5 \mathrm{mg}$ PSL or $\geq 5 \mathrm{mg}$ PSL) and GA, with adjustment for age, sex, BMI, Alb, Cre, and A1c. These factors were chosen a priori on the basis of their clinical significance and possible effect on GA. To evaluate multi-collinearity, the variance inflation factor (VIF) was calculated for each variable. VIF $>5$ was considered to indicate collinearity. The reliability of the model was internally validated using the bootstrap method. The optimism parameter in a calibration plot was calculated as the degree of overfitting, which was estimated using 150 sets of bootstrap sampling. We also conducted an analysis to assess the non-linear association between the dose of PSL as a continuous variable and plasma GA, in which PSL dose was modeled with the use of restricted cubic splines to allow for non-linear association. All statistical analyses were performed using $\mathrm{R}$ statistical software (version 3.5.1; available at http://www. rproject.org) and the "rms" package.

\section{RESULTS}

\section{Subject Characteristics}

A total of 48 patients (19 men and 29 women) were included in the final analysis (Table 2). Twenty-one patients had been taking $<5 \mathrm{mg}$ PSL per day and 27 had been taking $\geq 5 \mathrm{mg}$ PSL per day. The characteristics of the patients are shown in Table 1 . Age, gender, BMI, $\mathrm{Hb}$, and plasma A1c and Cre were similar between the two groups (Table 2), but the group taking $\geq 5 \mathrm{mg}$ PSL tended to have lower plasma Alb (Table 2).

\section{High Dose PSL Lowers Plasma GA Adjusted for Age, Sex BMI, Alb, and Cre}

To determine whether prednisolone use influenced GA concentration, we performed multiple linear regression analysis on plasma GA (Table 3). The relationship between the dose of PSL and GA was significant $(p=0.03)$ after adjustment for age, sex, BMI, Alb, Cre, and A1c. The $\geq 5 \mathrm{mg}$ PSL group had a lower GA $[\beta=-1.36 ; 95 \%$ confidence interval $(\mathrm{CI})-2.59$ to -0.14 ; Table 3, Fig. 1]. There was an approximate 0.06 increase in GA per 1 year increase in age $(\beta=0.06 ; 95 \%$ CI $0.03-0.09$; $p<0.001$; Table 2). There was also a trend towards GA being 0.22 lower in men $(\beta=-0.22 ; 95 \%$ CI -1.80 to $1.37 ; p=0.784$, Table 3, Fig. 2). An increase of 1 unit of BMI was associated with an approximate 0.14 reduction in GA $(\beta=-0.14 ; 95 \%$ CI -0.28 to -0.01 ; $p=0.042$, Table 3 ). In addition, Alb tended to be about 0.4 higher in patients taking high- 
Table 2 Baseline clinical characteristics of 48 subjects

\begin{tabular}{llll}
\hline & Total $(\boldsymbol{n}=\mathbf{4 8})$ & PSL $<\mathbf{5 ~} \mathbf{~ m g}(\boldsymbol{n}=\mathbf{2 1})$ & PSL $\geq \mathbf{5 ~} \mathbf{~ g}(\boldsymbol{n}=\mathbf{2 7})$ \\
\hline Age & $57.5[43.8-72.0]$ & $62.0[46.0-70.0]$ & $57.0[36.5-72.5]$ \\
Male sex (\%) & $19(39.6)$ & $8(38.1)$ & $11(31.2)$ \\
BMI & $23.38[20.22-24.48]$ & $23.37[20.06-26.93]$ & $23.38[20.43-23.83]$ \\
Alb & $4.2[4.0-4.3]$ & $4.2[4.1-4.4]$ & $4.1[4.0-4.2]$ \\
Cre & $0.63[0.55-0.74]$ & $0.66[0.59-0.75]$ & $0.60[0.53-0.73]$ \\
HbAlc & $5.9[5.6-6.4]$ & $5.7[5.5-6.0]$ & $6.0[5.7-6.6]$ \\
\hline
\end{tabular}

Table 3 Multiple linear regression analysis of GA

\begin{tabular}{lcccr}
\hline & Coefficient & $\mathbf{9 5 \% ~ L C I ~}$ & $\mathbf{9 5 \%}$ UCI & $p$ value \\
\hline Dose of PSL & -1.36 & -2.59 & -0.14 & 0.03 \\
Age & 0.06 & 0.03 & 0.09 & $<0.001$ \\
Male sex (\%) & -0.22 & -1.80 & 1.37 & 0.784 \\
BMI & -0.14 & -0.28 & -0.01 & 0.042 \\
ALB & 0.40 & -2.10 & 2.90 & 0.749 \\
CRE & 1.59 & -3.74 & 6.91 & 0.55 \\
Alc & 1.40 & 0.38 & 2.42 & 0.008 \\
\hline
\end{tabular}

dose PSL ( $\beta=0.4 ; 95 \%$ CI -2.1 to $2.9 ; p=0.749$; Table 3$)$ and Cre tended to be about 1.59 higher $(\beta=1.59 ; 95 \%$ CI -3.74 to $6.91 ; p=0.55$; Table 3). An increase of 1 unit of A1c was associated with an approximate 1.4 increase in GA ( $\beta=1.4$; 95\% CI 0.38-2.42; $p=0.008$; Table 3$)$.

Box plots of the predicted GA value, adjusted for covariance, are shown in Fig. 1. The adjusted $R^{2}$ was 0.518 , suggesting that the regression model could explain differences in GA well. The VIFs suggested that collinearity was not present among the variables (the VIFs were 1.35 for age, 2.30 for sex, 1.09 for BMI, 1.33 for Alb, 2.26 for Cre, and 1.49 for A1c.) The optimism parameter for the regression model was 0.09 , meaning that the degree of overfitting was $9.2 \%$; thus, there was no evidence of overfitting. Finally, the dose of PSL as a continuous variable was negatively correlated $(p=0.003)$ and weakly non-linearly associated $(p=0.46)$ with GA adjusted for age, sex, BMI, Alb, Cre, and A1c (Fig. 2), indicating that plasma GA decreases as the dose of PSL increases.

\section{DISCUSSION}

In this study, we investigated whether steroid administration affects GA. Multiple linear regression analysis showed that PSL dose, age, BMI, and A1c significantly correlated with plasma GA. After adjustment of GA for age, sex, $\mathrm{BMI}$, Cre, and A1c, it was higher in patients taking $5 \mathrm{mg}$ PSL than in those taking $<5 \mathrm{mg}$ PSL. Therefore, in subjects undergoing highdose ( $\geq 5 \mathrm{mg}$ ) PSL therapy, it is possible that the use of GA may be associated with a underestimation of the severity of hyperglycemia.

This study is the first to reveal that high-dose PSL lowers GA, although it was a small retrospective study. A previous study reported that 


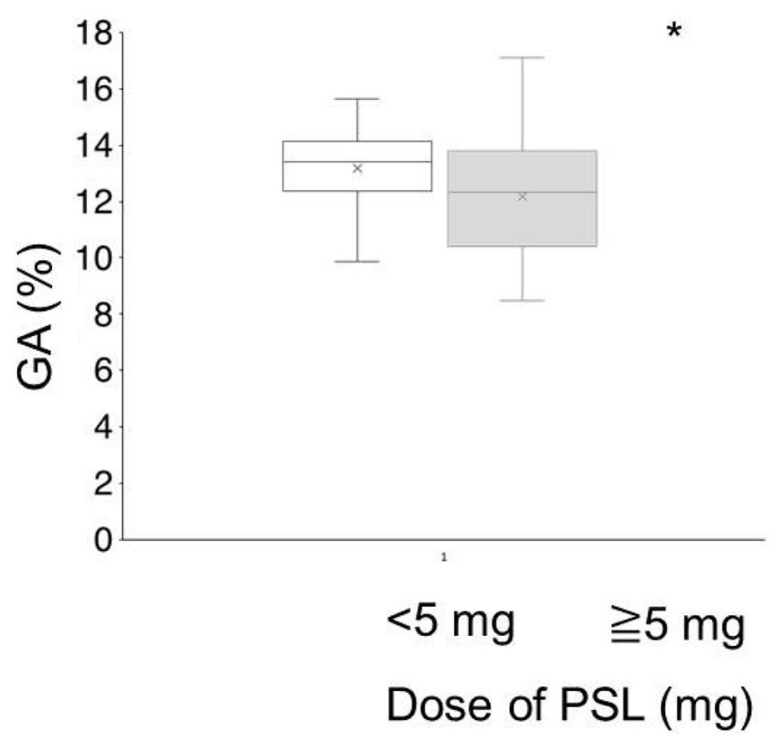

Fig. 1 Glycated albumin adjusted for age, sex, body mass index, creatinine, albumin, and glycated hemoglobin A1c is lower in patients taking $\geq 5 \mathrm{mg}$ prednisolone. Multiple linear regression analysis of the effect of prednisolone (PSL) dose on glycated albumin (GA) adjusted for age 57.5, sex $=$ M, BMI 23.38, plasma albumin (Alb) 4.2, plasma creatinine (Cre) 0.625 , and hemoglobin Alc 5.9. ${ }^{*} p<0.05 . n=21$ and 24 in the $<5 \mathrm{mg}$ PSL and $\geq 5 \mathrm{mg}$ PSL groups, respectively

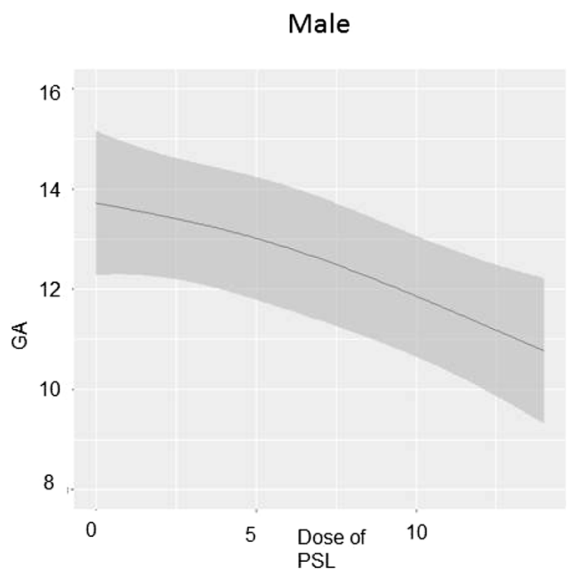

Fig. 2 Correlation between plasma glycated albumin and prednisolone dose. A multivariable linear regression model was used to evaluate the associations between dose of prednisolone (PSL) (as a continuous variable) and glycated hemoglobin (GA) adjusted for age, sex, body mass index (BMI), plasma albumin (Alb), plasma creatinine (Cre), and glycated hemoglobin Alc (A1c). The solid line
GA in patients with Cushing's syndrome is lower than in patients with type 2 DM [9], and a patient treated with $20 \mathrm{mg}$ prednisolone has also been described who exhibited GA-to-A1c ratios that were persistently lower (1.49-1.80) than the ratio normally present in type $2 \mathrm{DM}$ patients (2.5) [8]. It is known that alterations in albumin concentration can affect GA, and glucocorticoids upregulate both protein synthesis and degradation, thereby increasing protein turnover [10]. In fact, plasma albumin in patients taking $\geq 5 \mathrm{mg}$ PSL was significantly lower than that in those taking $<5 \mathrm{mg}$ PSL. When it is also considered that plasma GA in patients with nephrotic syndrome and hyperthyroidism is lower than would be expected according to the degree of glycemic control $[11,12]$, it is likely that high-dose PSL causes a reduction in GA as a result of altered albumin turnover.

The use of glucocorticoids can been associated with the development of osteoporosis, osteonecrosis, cataracts, hyperglycemia, coronary heart disease, and cognitive impairment, but the minimum dose required to induce these adverse effects has been shown to be about $5 \mathrm{mg}$

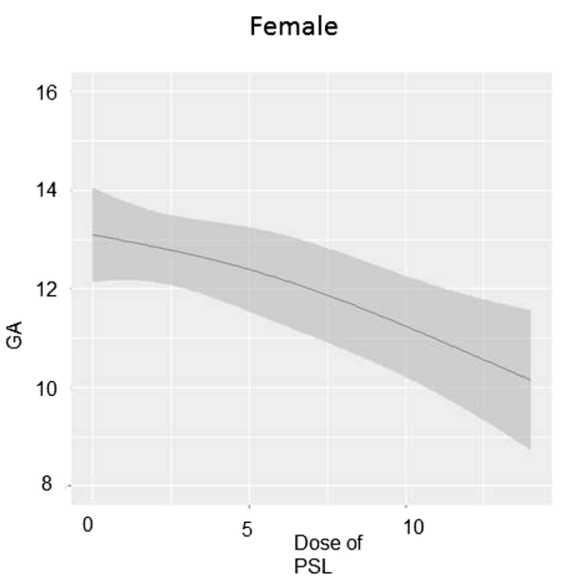

indicates the predicted GA after adjustment for age 57.5, sex $=$ male or female, BMI 23.38, Alb 4.2, Cre 0.625, A1c 5.9. The gray band indicates the $95 \%$ confidence interval for the regression line 
PSL per day $[13,14]$. Therefore, high-dose PSL is often defined as $\geq 5 \mathrm{mg}$ PSL $[13,14]$. Moreover, the incidence of DM in patients taking $\geq 5 \mathrm{mg}$ PSL was five times higher than in patients taking $<5 \mathrm{mg}$ PSL [15]. Therefore, we divided the subjects in this study into those taking $<5 \mathrm{mg}$ PSL and those taking $\geq 5 \mathrm{mg}$ PSL. Our data show that the use of GA as an indicator of glycemic status in patients taking high doses of steroids may be associated with an underestimation of the severity of hyperglycemia.

It has previously been reported that A1c, age, and BMI significantly affect plasma GA levels [16-19]. Because A1c also indicates the severity of recent glycemia, it is closely correlated with GA $[17,18]$, and the relationship has been described by $\mathrm{HbA} 1 \mathrm{c}=0.216 \times \mathrm{GA}+2.978$ $\left[R^{2}=0.5882, p<0.001\right]$ [18]. GA increases with age from infancy to adulthood and therefore age-adjusted GA can more accurately reflect glycemic status $[19,20]$. Most obese individuals are insulin resistant, which can be associated with postprandial hyperglycemia, which tends to increase GA rather than A1c [7, 21, 22], such that, for example, the GA-to-A1c ratio is often higher in patients with type $1 \mathrm{DM}[7,21]$. Moreover, the risk of steroid-induced DM is greater in individuals with a higher BMI [23]. However, our data show that GA is negatively correlated with BMI, which is consistent with many previous reports [24-26]. Finally, other authors have speculated that greater protein turnover and inflammation may contribute to the observed GA levels in obese subjects [26]. Although the mechanism has not been elucidated in this study, A1c, age, and BMI have been shown to affect GA concentration.

This study had a number of limitations. Firstly, it was not a randomized study, it was small, and conducted only in one hospital; therefore, we are mindful that there is a risk of selection bias. To further investigate the effects of PSL dose on GA, a prospective study (such as a cohort study) is required. Secondly, the range of A1c concentrations was limited to $5.0-7.5 \%$; therefore, conclusions regarding GA can only be drawn for patients with A1c values within this range. Thirdly, we used A1c as a marker of glycemic control, rather than multiple or continuous peripheral blood glucose measurements, which can provide a more accurate picture of glycemic control, but would not be covered by health insurance in Japan. Finally, we only studied subjects with connective tissue diseases and not healthy individuals, for which we would not have had the opportunity to measure A1c and GA; therefore, we could not compare data obtained from diseased subjects with healthy subjects.

\section{CONCLUSION}

We have demonstrated that high doses of PSL, as well as age, high BMI, and high A1c, are associated with relatively low GA concentrations compared with the severity of hyperglycemia. Thus, when high-dose ( $\geq 5 \mathrm{mg}$ ) PSL is administered to patients with CTDs, the use of GA might be associated with an underestimation of the degree of glycemic control.

\section{ACKNOWLEDGEMENTS}

We thank all the patients included in this study.

Funding. No funding or sponsorship was received for this study or the publication of this article. The article processing charges were funded by the authors.

Editorial Assistance. We also thank Charles Allan, PhD, and Mark Cleasby, PhD, from Edanz Group (www.edanzediting.com/ac) for editing drafts of this manuscript. This was funded by the authors.

Authorship. All named authors meet the International Committee of Medical Journal Editors (ICMJE) criteria for authorship for this article, take responsibility for the integrity of the work as a whole, and have given their approval for this version to be published.

Disclosures. Masami Mizuno, Katsumi Iizuka, Takuma Ishihara, Shusaku Fukaya, Shunji Yoshida, and Jun Takeda declare that they have nothing to disclose. 
Compliance with Ethics Guidelines. All procedures performed in studies involving human participants were in accordance with the ethical standards of the institutional and/or national research committee and with the 1964 Declaration of Helsinki and its later amendments or comparable ethical standards. This research used non-identifiable data obtained by the treating physicians, and therefore on the basis of the decision of the local ethics committee of our university (Approval No. 28-139, approval date 2016/8/3), informed consent was not required. This study was not interventional but retrospective and observational, and therefore did not have a trial registration ID.

Data Availability. The datasets collected and/or analyzed during the current study are available from the corresponding author on reasonable request.

Open Access. This article is distributed under the terms of the Creative Commons Attribution-NonCommercial 4.0 International License (http://creativecommons.org/licenses/ by-nc/4.0/), which permits any noncommercial use, distribution, and reproduction in any medium, provided you give appropriate credit to the original author(s) and the source, provide a link to the Creative Commons license, and indicate if changes were made.

\section{REFERENCES}

1. Clore JN, Thurby-Hay L. Glucocorticoid-induced hyperglycemia. Endocr Pract. 2009;15:469-74.

2. Ruiz-Irastorza G, Danza A, Khamashta M. Glucocorticoid use and abuse in SLE. Rheumatology (Oxford). 2012;51:1145-53.

3. Tamez-Pérez HE, Quintanilla-Flores DL, RodríguezGutiérrez R, González-González JG, Tamez-Peña AL. Steroid hyperglycemia: prevalence, early detection and therapeutic recommendations: a narrative review. World J Diabetes. 2015;6:1073-81.

4. Radin MS. Pitfalls in hemoglobin A1c measurement: when results may be misleading. J Gen Intern Med. 2014;29:388-94.
5. American Diabetes Association. 6. Glycemic targets: standards of medical care in diabetes-2018. Diabetes Care. 2018;41:S55-S64.

6. Takahashi S, Uchino H, Shimizu T, et al. Comparison of glycated albumin (GA) and glycated hemoglobin (HbA1c) in type 2 diabetic patients: usefulness of GA for evaluation of short-term changes in glycemic control. Endocr J. 2017;54:139-44.

7. Koga M, Murai J, Morita S, Saito H, Kasayama S. Comparison of annual variability in $\mathrm{HbA} 1 \mathrm{c}$ and glycated albumin in patients with type 1 vs. type 2 diabetes mellitus. J Diabetes Complicat. 2013;27:211-3.

8. Iizuka K, Kato T, Mizuno M, Takeda J. A discrepancy between plasma glycated albumin and HbA1c levels in a patient with steroid-induced diabetes mellitus. BMJ Case Rep. 2016;2016. https://doi.org/10.1136/ bcr-2016-214788.

9. Kitamura T, Otsuki M, Tamada D, et al. Serum albumin-adjusted glycated albumin is an adequate indicator of glycemic control in patients with Cushing's syndrome. Clin Biochem. 2014;47:279-82.

10. Rothschild MA, Schreiber SS, Oratz M, McGee HL. The effects of adrenocortical hormones on albumin metabolism studied with albumin-I131. J Clin Invest. 1958;37:1229-35.

11. Okada T, Nakao T, Matsumoto H, et al. Influence of proteinuria on glycated albumin values in diabetic patients with chronic kidney disease. Intern Med. 2011;50:23-9.

12. Koga M, Murai J, Saito H, Matsumoto S, Kasayama S. Effects of thyroid hormone on serum glycated albumin levels: study on non-diabetic subjects. Diabetes Res Clin Pract. 2009;84:163-7.

13. Saag KG, Koehnke R, Caldwell JR, et al. Low dose long-term corticosteroid therapy in rheumatoid arthritis: an analysis of serious adverse events. Am J Med. 1994;96:115-23.

14. Da Silva JA, Jacobs JW, Kirwan JR, et al. Safety of low dose glucocorticoid treatment in rheumatoid arthritis: published evidence and prospective trial data. Ann Rheum Dis. 2006;65:285-93.

15. Gurwitz JH, Bohn RL, Glynn RJ, et al. Glucocorticoids and the risk for initiation of hypoglycemic therapy. Arch Intern Med. 1994;154:97-101.

16. Wang F, Ma X, Hao Y, et al. Serum glycated albumin is inversely influenced by fat mass and visceral adipose tissue in Chinese with normal glucose tolerance. PLoS One. 2012;7:e51098. 
17. Testa R, Ceriotti F, Guerra E, et al. Glycated albumin: correlation to HbA1c and preliminary reference interval evaluation. Clin Chem Lab Med. 2017;55:e31-3.

18. Inoue $\mathrm{K}$, Tsujimoto $\mathrm{T}$, Yamamoto-Honda $\mathrm{R}$, et al. A newer conversion equation for the correlation between HbA1c and glycated albumin. Endocr J. 2014;61:553-60.

19. Suzuki S, Koga M, Niizeki N, et al. Age-adjusted glycated albumin: a more robust parameter to establish glycaemic control in neonatal diabetes mellitus. Ann Clin Biochem. 2014;51:602-5.

20. Furuya A, Suzuki S, Oshima M, et al. Age-adjusted glycated albumin at diagnosis is more correlated with the product of age and plasma glucose than plasma glucose alone in patients with neonatal diabetes mellitus. J Clin Lab Anal. 2016;30:1086-91.

21. Matsumoto H, Murase-Mishiba Y, Yamamoto N, et al. Glycated albumin to glycated hemoglobin ratio is a sensitive indicator of blood glucose variability in patients with fulminant type 1 diabetes. Intern Med. 2012;51:1315-21.
22. Lee EY, Lee BW, Kim D, et al. Glycated albumin is a useful glycation index for monitoring fluctuating and poorly controlled type 2 diabetic patients. Acta Diabetol. 2011;48:167-72.

23. Ariza-Andraca CR, Barile-Fabris LA, Frati-Munari AC, Baltazar-Montufar P. Risk factors for steroid diabetes in rheumatic patients. Arch Med Res. 1998;29:259-62.

24. Huh JH, Kim KJ, Lee B-W, et al. The relationship between BMI and glycated albumin to glycated hemoglobin (GA/A1c) ratio according to glucose tolerance status. PLoS One. 2014;9:e89478.

25. Koga M, Hirata T, Kasayama S, Ishizaka Y, Yamakado M. Body mass index negatively regulates glycated albumin through insulin secretion in patients with type 2 diabetes mellitus. Clin Chim Acta. 2015;438:19-23.

26. Koga M, Matsumoto S, Saito H, Kasayama S. Body mass index negatively influences glycated albumin, but not glycated hemoglobin, in diabetic patients. Endocr J. 2006;53:387-91. 